\title{
Anesthetic management of a patient with dilated cardiomyopathy for fracture femur surgery: a case report
}

\begin{abstract}
Dilated cardiomyopathy (DCMP), especially alcoholic cardiomyopathy is a myocardial disease and is challenging for perioperative management. As anesthesiologist one should be aware of its pathophysiology, clinical features, diagnostic evaluations and the treatment modalities. Moreover, meticulous planning is required for safe and uneventful anesthesia. There is limited literature on anesthetic management of patients with alcoholic cardiomyopathy undergoing orthopedic surgery under combined spinal epidural anesthesia. We report a case of dilated cardiomyopathy scheduled for dynamic hip screw surgery for fracture neck femur.
\end{abstract}

Keywords: dilated cardiomyopathy, alcoholic, anesthesia
Volume 2 Issue I - 2015

\author{
Nishkarsh Gupta, Rakesh Garg,Anju Gupta, \\ Sachidanand Jee Bharati,Vinod Kumar \\ Chanpadia \\ Department of Anesthesiology, RML Hospital, India
}

Correspondence: Nishkarsh Gupta, Department of Anesthesiology, RML Hospital, India, Tel 9868398338 , Email drnishkarsh@rediifmmail.com

Received: January 07, 2015 | Published: January 21, 2015
Abbreviations: DCMP, dilated cardiomyopathy; NIBP, noninvasive blood pressure; ECG, electrocardiograms; CHF, congestive heart failure; PCWP, Pulmonary Capillary Wedge Pressure

\section{Introduction}

Dilated cardiomyopathy (DCMP) is a myocardial disease of varied causes characterized by dilatation of one or both the ventricles, impaired myocardial contractility, decreased cardiac output and increased ventricular filling pressures. ${ }^{1}$ Alcoholic cardiomyopathy is the most common secondary cardiomyopathy. The typical presentation of alcoholic cardiomyopathy is in the 4-6 decade of life with a male predilection. ${ }^{2,3}$ The anesthetic management of these patients is quite challenging. As anesthesiologist one should be aware of its pathophysiology, clinical features, diagnostic evaluations and the treatment modalities. Moreover, meticulous planning is required for safe and uneventful anesthesia. There is limited literature on anesthetic management of patients with alcoholic cardiomyopathy undergoing orthopedic surgery under combined spinal epidural anesthesia. ${ }^{4,5}$ We report a case of dilated cardiomyopathy scheduled for dynamic hip screw surgery for fracture neck femur.

\section{Case report}

A 49year old man sustained sub trochanteric femur fracture due to fall under alcohol intoxication and was scheduled for dynamic hip screw surgery. He was a known case of diabetes mellitus since 10years and taking oral metformin, He was known alcoholic since 12 years. On examination, patient had dyspnea at rest; his heart rate was 76/ min and regular. The blood pressure was $114 / 74 \mathrm{mmHg}$. Preoperative 12 lead electrocardiograms showed sinus tachycardia with left bundle branch block. Chest x-ray revealed cardiomegaly with CT ratio of 0.7 . Echocardiography showed hypokinetic anterior wall with ejection fraction of $30 \%$, with trace mitral regurgitation and grade 3 diastolic dysfunction. Dobutamine Stress echocardiogram was negative for inducible ischemia. All the biochemical and hematological investigations were within the normal limits. His symptoms were controlled with oral carbidilol $25 \mathrm{mg}$ twice a day, oral ramipril $2.5 \mathrm{mg}$ once a day and subcutaneous deltaparin 5000 units twice daily. High risk consent was obtained in view of poor cardiovascular status (American society of Anesthesiology status III) and the patient was planned to receive combined spinal and epidural anesthesia (CSE).
In the operating room standard monitors including non-invasive blood pressure (NIBP), arterial oxygen saturation (SpO2) and five lead electrocardiograms (ECG) were attached. Under all aseptic precautions and local anesthesia, an arterial line (left radial) and central line (right Internal jugular) were established for continuous arterial and central venous pressures. After taking all aseptic precautions, an $18 \mathrm{G}$ epidural needle was introduced at L3-4 space in the left lateral position and a 26 $\mathrm{G}$ spinal needle was introduced through it. Intrathecally $2.5 \mathrm{mg}(0.5 \mathrm{ml}$ of $0.5 \%$ ) hyperbaric bupivacaine with fentanyl $25 \mathrm{mcg}$ (total volume $=1$ $\mathrm{ml}$ ) was administered. Thereafter, epidural catheter threaded into the same space and fixed at $7 \mathrm{~cm}$. The patient was subsequently placed in the supine position with slightly head up position. A T10 sensory block to pin-prick was achieved. Ringer lactate was given to maintain CVP $6-8 \mathrm{~cm}$ water. The surgery lasted 80 minutes and there was a blood loss of $400 \mathrm{~mL}$. A total of $1000 \mathrm{~mL}$ of normal saline and one unit packed red blood cells were given. The patient remained hemodynamically stable throughout the surgery. After surgery the patient was shifted to postoperative ward for monitoring. The patient received $3 \mathrm{mg}$ epidural morphine in $5 \mathrm{~mL}$ normal saline for postoperative analgesia. The subsequent postoperative course was uneventful. The patient was shifted to intensive care unit in the immediate postoperative period and the hemodynamic monitoring was continued (ECG, arterial blood pressure, CVP). The blood pressure was maintained, there were no complaint of chest pain, sweating or dysnoea. The patient was given epidural morphine $3 \mathrm{mg}$ in $5 \mathrm{~mL}$ normal saline two times a day for analgesia. Single dose subcutaneous delta parin in the night was restarted on second postoperative day. Epidural catheter was removed on the morning of third postoperative day. The patient was discharged subsequently and followed up in the outpatient clinic.

\section{Discussion}

Anesthetic management of patients with cardiomyopathy can be challenging and may be associated with high morbidity and mortality. ${ }^{1}$ DCMP is defined as deterioration in cardiac function due to dilatation and impaired contraction of the left or both ventricles. The prevalence is $920 / 100000$. The causes are varied and may be ischemic or non ischemic. The ischemic type is related to atherosclerosis and ischemic heart disease. ${ }^{2}$ The non ischemic type may be secondary to infections, chemotherapeutic agents, alcohol abuse or during the per partum period. Though the mechanism of alcohol mediated cardiac 
damage remains unclear, the most evidence in the literature suggests a direct toxic result of ethanol or its metabolites on the heart. The other theories like nutritional deficiencies (e.g., thiamine deficiency) and associated co morbidities (e.g., hypertension) may have a role in some of the patients. Polymorphism of gene encoding the alcohol dehydrogenase $(\mathrm{AIDH} 2 * 2)$ also increases the predisposition to development of dilated cardiomyopathy. ${ }^{1,2}$

The onset of symptoms is usually insidious and includes dysnoea, orthopnea, paroxysmal nocturnal dysnoea, chest discomfort, fatigue, palpitations, dizziness, syncope, anorexia etc. Patients with an ejection fraction of $<25 \%$, a hypokinetic and dilated left ventricle, pulmonary capillary wedge pressure $(\mathrm{PCWP})>20 \mathrm{mmHg}$, cardiac index $<2.5 \mathrm{~L} / \mathrm{min} / \mathrm{m},{ }^{2}$ systemic hypotension, pulmonary hypertension and an increased CVP have poor prognosis. The management of a patient with DCMP is most commonly complicated by progressive congestive heart failure (CHF) and malignant arrhythmias (the most common cause of death in DCM) ${ }^{1,2}$ The treatment aims at improving systolic function by medicines, biventricular pacing, cardio plasty or cardiac transplant.

The ideal goals of anesthetic management include avoidance of drug induced myocardial depression, maintenance of normovolemia and prevention of increased ventricular after load. ${ }^{3}$ Keeping these goals in mind we had planned CSE in our patient. During general anesthesia dose dependent myocardial depression may occur with use of volatile anesthetics, administration of opioid, nitrous oxide or benzodiazepine. Patient may also be vulnerable to drug over dosage due to slow circulation time. Moreover stress response to intubation and extubation along with decreased preload due to positive pressure ventilation may also be deleterious to the patient. Therefore regional technique may be preferred over general anesthesia in selected patients. Spinal anesthesia alone can cause a precipitous fall in blood pressure and does not provide postoperative analgesia. Epidural alone can also have unpredictable effect. So, they were not considered by us, rather we decided to go ahead with sequential CSE technique.

The combined low dose spinal with epidural anesthesia decreases after load, prevented myocardial depression. Fluid management is also critical and preloading the patient in the preoperative period is not desirable because it may precipitate a congestive heart failure. In our case fluid overloading was prevented by titrate the fluids to maintainin a CVP of $8-10 \mathrm{~cm} \mathrm{H} 2 \mathrm{O}$. We chose to do a sequential CSE technique which not only provided us with a rapid onset but also gave the flexibility of further extension of the block with the epidural catheter. Also this technique has been found to produce better analgesia and muscle relaxation with decreased associated hypotension. ${ }^{4,5}$ Moreover, we used a very small dose of local anesthetic intrathecal in order to minimize hypotension. Subsequently, $4 \mathrm{~mL}$ (2 $+2 \mathrm{~mL}$ ) of local anaesthetic was sequentially used via the epidural catheter to achieve a sensory level of T 10 . Fluids were given carefully maintain a CVP of $8 \mathrm{~cm}$ water. Invasive monitoring like invasive blood pressure monitoring through arterial line and central venous pressure monitoring were used to facilitate early recognition of blood pressure changes and to guide fluid therapy and maintain normovolemia.
Although the management of such a patient can be quite challenging to the anesthetist, this patient had a smooth intra op, and postoperative period, due to vigilant monitoring, prompt treatment of complications and a good anesthetic plan of action. To the best of our knowledge, this is one of the first case reports the DHS has been done under sequential CSE in a patient with severe DCMP.

\section{Conclusion}

In conclusion, the anesthetic management of a patient with DCMP undergoing non cardiac surgery is challenging and may be associated with high mortality. As anesthesiologist a careful anesthesia planning, pre-operative assessment, interaoperative and postoperative management can help in reducing the mortality. CSE using lowdose intrathecal bupivacaine and fentanyl with sequential epidural bupivacaine provided the advantages of spinal and epidural blockade, whilst avoiding some of their respective limitations. This sequential CSE technique may be particularly helpful in high risk cardiac disease patients in whom a slower onset sympathetic blockade is required. Thus, low-dose sequential CSE can be a safe alternate to achieve good anesthesia with impressive cardiovascular stability.

\section{Acknowledgments}

None.

\section{Funding details}

None.

\section{Conflicts of interest}

Authors declare that there is no conflict of interest.

\section{References}

1. Stoelting RK, Dierdorf SF. Cardiomyopathy. In:Stoelting RK (Ed.), Anaesthesia and Coexisting Disease. (3rd edn), Churchill Livingstone, New York, USA, 1993; pp. 97-102.

2. Dec WG, Fuster V. Idiopathic dilated Cardiomyopathy. $N$ Engl $J$ Med. 1994;331(23):1564-1575.

3. Kaur H, Khetarpal R, Aggarwal S. Dilated cardiomyopathy:an anaesthetic challenge. J Clin Diagn Res. 2013;7(6):1174-1176.

4. Hamlyn EL, Douglass CA, Plaat F, et al. Low-dose sequential combined spinal-epidural:An anaesthetic technique for caesarean section in patients with significant cardiac disease. Int $J$ Obstet Anesth. 2005;14(4):355-361

5. Hamdani GA, Chohan U, Zubair NA. Clinical usefulness of sequential combined spinal epidural anaesthesia in high risk geriatric patients for major orthopaedic surgery. $J$ Anaesth Clin Pharmacol. 2002;18(2):163-166. 